\title{
XMM-Newton long-look observation of the narrow-line Seyfert 1 galaxy PKS 0558-504
}

\section{Timing analysis}

\author{
I. E. Papadakis ${ }^{1,2}$, W. Brinkmann ${ }^{3}$, M. Gliozzi ${ }^{4}$, and C. Raeth ${ }^{3}$ \\ 1 Physics Department, University of Crete, PO Box 2208, 71003 Heraklion, Crete, Greece \\ e-mail: jhep@physics.uoc.gr \\ 2 IESL, Foundation for Research and Technology, 711 10, Heraklion, Crete, Greece \\ 3 Max-Planck-Institut für extraterrestrische Physik, Giessenbachstrasse, 85740 Garching, Germany \\ ${ }^{4}$ George Mason University, Department of Physics and Astronomy, MS 3F3, 4400 University Dr., Fairfax, VA 22030-4444, USA
}

Received 1 April 2010 / Accepted 25 May 2010

\section{ABSTRACT}

Context. PKS 0558-504 has been observed repeatedly by XMM-Newton as a calibration and performance verification target. In this work, we present results from the power spectral analysis using data from a long XMM-Newton observation and archival RXTE data. Aims. To search for periodic and/or quasi-periodic signals, to estimate the power spectrum over a broad range which covers almost six decades in frequency, and to compare the variability in the ultraviolet (UV), soft and hard X-ray bands.

Methods. We used the XMM-pn soft and hard band light curves to compute the high frequency power spectrum (PSD) and search for periodicities. We also used an archival, long RXTE light curve to constrain the power spectrum at low frequencies, and the XMMOM light curve to estimate the UV PSD. We also used the results from the time-resolved spectral studies we presented in a recent paper to compare in more detail the X-ray, "soft-excess" and UV variations.

Results. We did not detect any significant periodic or quasi-periodic signals. The soft band power spectral slope is steeper than the hard band PSD slope, but consistent within the 1- $\sigma$ uncertainties. The broad frequency band, 2-10 keV PSD follows a power-law like shape of an index $\sim 0.5$ up to a break frequency at $\sim 7 \times 10^{-6} \mathrm{~Hz}$ (which corresponds to a time scale of 1.7 days). At higher frequencies, the PSD steepens to a slope of $\sim 2.2$. The OM power spectrum has a significantly smaller amplitude than the X-ray and the soft-excess PSDs.

Conclusions. The similarity between the PSD shape of PKS 0558-504 and other radio-quiet Seyferts 1 suggests a common variability (and emission) mechanism. Consequently any jet emission in the X-ray band should be unimportant in this source. Using the scaling relations between PSD break time scales and black hole mass/bolometric luminosity, we estimated a black hole mass of $\sim 2 \times 10^{8} M_{\odot}$. Power spectral analysis of the light curves which resulted from the time-resolved spectroscopic study of the same data set indicates that the intrinsic power spectrum may steepen with increasing energy, contrary to what is currently believed for typical Seyferts. When scaled appropriately, the $\mathrm{OM}$ and soft-excess light curves show similar variability patterns. They may originate from the accretion disc, at a distance of $\sim 20$ and 5 Schwarzschild radii, respectively, and their variations could be due to accretion rate variations, which operate on the local viscosity time scale and propagate inwards.

Key words. galaxies: active - galaxies: Seyfert - X-rays: galaxies - galaxies: individual: PKS 0558-504

\section{Introduction}

Narrow-line Seyfert 1 galaxies (NLS1) have been rarely found to be radio loud. Recent studies (Komossa et al. 2006) have shown that the radio loud fraction of the NLS1 population is less than $\sim 7 \%$. PKS 0558-504 $\left(z=0.1372, m_{\mathrm{B}}=14.97\right)$ belongs to this group of rare objects. It is one of the best studied radioloud NLS1 galaxies and it has repeatedly shown large amplitude $\mathrm{X}$-ray flux variations in the past.

GINGA observations (Remillard et al. 1991) detected an increase in the X-ray flux by $67 \%$ in 3 min, implying that the apparent luminosity must be enhanced by relativistic beaming. The six days long ROSAT All Sky Survey light curve of September 1990, and a series of pointed HRI observations in April 1998, showed count rate variations by a factor of 2 in less than one day (Gliozzi et al. 2000). The most extreme value of the luminosity variations found during the observations, $\Delta L / \Delta t=$ $1.12 \times 10^{42} \mathrm{erg} \mathrm{s}^{-2}$, implied a lower limit of $\eta>0.54$, where $\eta$ is the efficiency with which matter is converted to energy. This limit exceeds even the theoretical maximum for accretion onto a maximally rotating Kerr black hole. Dramatic flux variations by a factor of 2 in $33 \mathrm{~min}$ and, perhaps, by $40 \%$ on a time scale as short as 2 min, were reported by Wang et al. (2001) from ASCA observations on January 31, 2000. These values implied an even higher value of $\eta=0.9 \pm 0.2$. PKS 0558-504 has been monitored regularly with $R X T E$ since 2005. Gliozzi et al. (2007) presented the results from the timing analysis of the first year of this monitoring campaign. They found that the X-ray flux typically changes by a factor of 1.5-2 on time scales of 10-30 days. A few extreme events, where the flux increases by a factor of $\sim 4$ in 3 days, were also observed.

The source has also been observed repeatedly by XMM-Newton as a calibration and performance verification (PV) target. Gliozzi et al. (2001) published the results from the timing analysis of some of the preliminary data of the commissioning/CalPV phase. The light curve showed persistent 
variability. Short term variations, characterized by smooth modulations with a typical time scale of $\sim 2.2 \mathrm{~h}$, were observed. The most extreme count rate variations found implied again a radiative efficiency slightly higher than the theoretical maximum for accretion onto a Schwarzschild black hole. An analysis of all PV phase and calibration observations performed by $X M M$-Newton up to orbit 341 with various instrumental settings was presented by Brinkmann et al. (2004). The authors noticed the presence of X-ray flares which seemed to be recurrent with a time scale of $\sim 24 \mathrm{ks}$. However, the statistical significance of the signal could not be assessed reliably due to the extreme sparsity of the data.

In September 2008, PKS 0558-504 was observed with $X M M-N e w t o n$ for five consecutive orbits. The results from the spectral analysis of these data have been presented in Papadakis et al. (2010) (Paper I, hereafter). The main objective of this long observation was to study its X-ray flux variability, and in particular, to investigate whether it exhibits any quasi-periodic signals. In this work, we present our main results based on the power spectrum (PSD) analysis of the XMM-Newton PN and OM light curves. In addition, we used propriety $R X T E$ data from the ongoing monitoring campaign of the source, to extend the PSD analysis to frequencies as low as $\sim(1 /$ year $)$. We also used some of the results from the time resolved spectral analysis presented in Paper I to investigate the variability properties of the X-ray power-law continuum in the soft and hard X-ray bands.

We start with the observational details and the data analysis in the next section. Section 3 presents our results from the PSD analysis of the XMM-Newton, and RXTE light curves. In Sect. 4 we summarize our results and discuss their implications.

\section{Observations and data analysis}

\subsection{The XMM-Newton observations}

PKS 0558-504 was observed with all instruments on board XMM-Newton for five consecutive orbits starting from September 09, 2008, 1:19 UT, and ending on September 16, 2008, 12:02 UT. The observation identifiers range from 0555170201 (XMM revolution 1602) to 0555170601 (XMM revolution 1606). In the following we will identify the individual observations by the last 3 digits (i.e. "obs-201", “obs-301”, etc.).

In this work we will use data from the PN camera only (Strüder et al. 2001), due to its superior statistical quality. The PN was operated in Small Window mode (SW) with thin filter. The data were processed using XMMSAS version 8.0. Source counts were accumulated from a rectangular box of $27 \times 26$ RAW pixels ( 1 RAW pixel $\sim 4.1^{\prime \prime}$ ) around the position of the source. Background data were extracted from a similar, source free, region on the chip. We selected single and double events (PATTERN $\leq 4$, FLAG $=0$ ).

We produced light curves in the $0.4-2 \mathrm{keV}$ and $2-10 \mathrm{keV}$ energy bands in $100 \mathrm{~s}$ bins (the "soft" and "hard" band light curves, hereafter). The data were screened for high background in both bands, and we excluded periods when the hard band background rate exceeded 0.25 counts $\mathrm{s}^{-1}$. High background activity affected the first 12.4 and $2.9 \mathrm{ks}$ of obs-201 and 301, and the data after $105,110,93.1$, and $123 \mathrm{ks}$ since the start of obs201, 301, 401 and 501, respectively. Background flaring activity affected obs-601 for two short periods during 22.5-27 ks and 86-88 ks after the start of the observation. The duration of the XMM-Newton light curves for each observation after the rejection of those times which were affected by high background,
Table 1. Light curve sampling parameters and variability amplitudes.

\begin{tabular}{lccc}
\hline \hline Light curve & $\begin{array}{c}T / \Delta t \\
(\mathrm{ks} / \mathrm{s})\end{array}$ & $\begin{array}{c}\text { Mean } \\
\left(\text { counts s}^{-1}\right)\end{array}$ & $\begin{array}{c}F_{\mathrm{var}} \\
(\%)\end{array}$ \\
& & $($ Soft/Hard band) & (Soft/Hard band) \\
\hline$X M M /$ obs-201 & $92.1 / 100$ & $7.25 / 0.94$ & $28.78 / 29.56$ \\
$X M M /$ obs-301 & $107 / 100$ & $7.18 / 0.90$ & $16.71 / 20.51$ \\
$X M M /$ obs-401 & $93.2 / 100$ & $10.04 / 1.34$ & $20.73 / 23.96$ \\
$X M M /$ obs-501 & $118.1 / 100$ & $8.26 / 1.13$ & $21.86 / 20.65$ \\
$X M M /$ obs-601 & $110.1 / 100$ & $10.59 / 1.39$ & $35.04 / 35.72$ \\
RXTE/2-10 keV & $1592 / 2$ (days) & 1.44 & 31.2 \\
$X M M / O M$ & $792.5 / 4.3(\mathrm{ks})$ & $1.1 \times 10^{-14 a}$ & 0.70 \\
\hline
\end{tabular}

Notes. ${ }^{(a)}$ in units of $\mathrm{erg} \mathrm{cm}^{-2} \mathrm{~s}^{-1} \AA^{-1}$.

the sampling time $\Delta t$, their mean count rates, and fractional root mean square variability amplitude, $F_{\text {var }}$, are listed in Table 1 . The first and second number listed in the third and fourth columns correspond to the soft and hard band light curves, respectively.

The $F_{\text {var }}$ values are corrected for the experimental noise, and their errors are $0.04 \%$ and $0.02 \%$ for the hard and soft band light curves, respectively. This estimate accounts for the measurement errors of the light curve points only, and has been calculated according to the prescription of Vaughan et al. (2003). On average, the soft band variability amplitude $\left(\bar{F}_{\text {var,soft }}=21.6 \pm 3.2 \%\right)$, is smaller than the variability amplitude of the hard band light curves $\left(\bar{F}_{\text {var,hard }}=26.1 \pm 2.6 \%\right)$, as is often the case with Seyfert galaxies.

The XMM-Newton soft and hard band light curves are plotted in Fig. 1. They look very similar, and the X-ray flux, in both bands, is highly variable. We observe substantial and continuous variability in all of them. However, the fast and very large amplitude events that were observed in the past are absent in the present XMM-Newton observations. To quantify the maximum $\Delta L / \Delta t$ variation in the hard band light curves (which exhibit the largest variability amplitude), we used the results from the time resolved spectroscopy reported in Paper I. In that paper, we slit the PN data in $2000 \mathrm{~s}$ long segments and fitted them with a power-law model. Using the best fit, $2-10 \mathrm{keV}$ flux measurements from these fits, we found that $[\Delta L(2-10 \mathrm{keV}) / \Delta t]_{\max }=1.2 \times 10^{41} \mathrm{erg} \mathrm{s}^{-2}$ during the $X M M-N e w t o n$ observations. Following Fabian (1979), this value leads to a lower limit of $\eta \geq 0.06$, which is not especially large, even for a non-rotating black hole.

There are just 6,1,2 and 5 points missing in the light curves of obs-201, 301, 401 and 501, caused by telemetry problems which reduced the "live time" of the detector to be less than $100 \mathrm{~s}$. The number of missing points in the obs-601 is higher, due to these telemetry problems and the presence of the two shortlived, background mini flares mentioned above. But even in this case, they account no more than $3 \%$ of the total number of points in the respective light curve. Since power spectrum analysis requires the use of evenly sampled light curves, we accounted for all missing points by linear interpolation between the bins adjacent to the gaps. We also added the appropriate Poisson noise in the interpolated values.

\subsection{The RXTE observations}

Since March 4, 2005, PKS 0558-504 has been observed with $R X T E$ approximately once every 2 days, for $\sim 1000-2000 \mathrm{~s}$ each time. In this work, we used data obtained until July 13, 2009. The observations were carried out with the Proportional Counter 


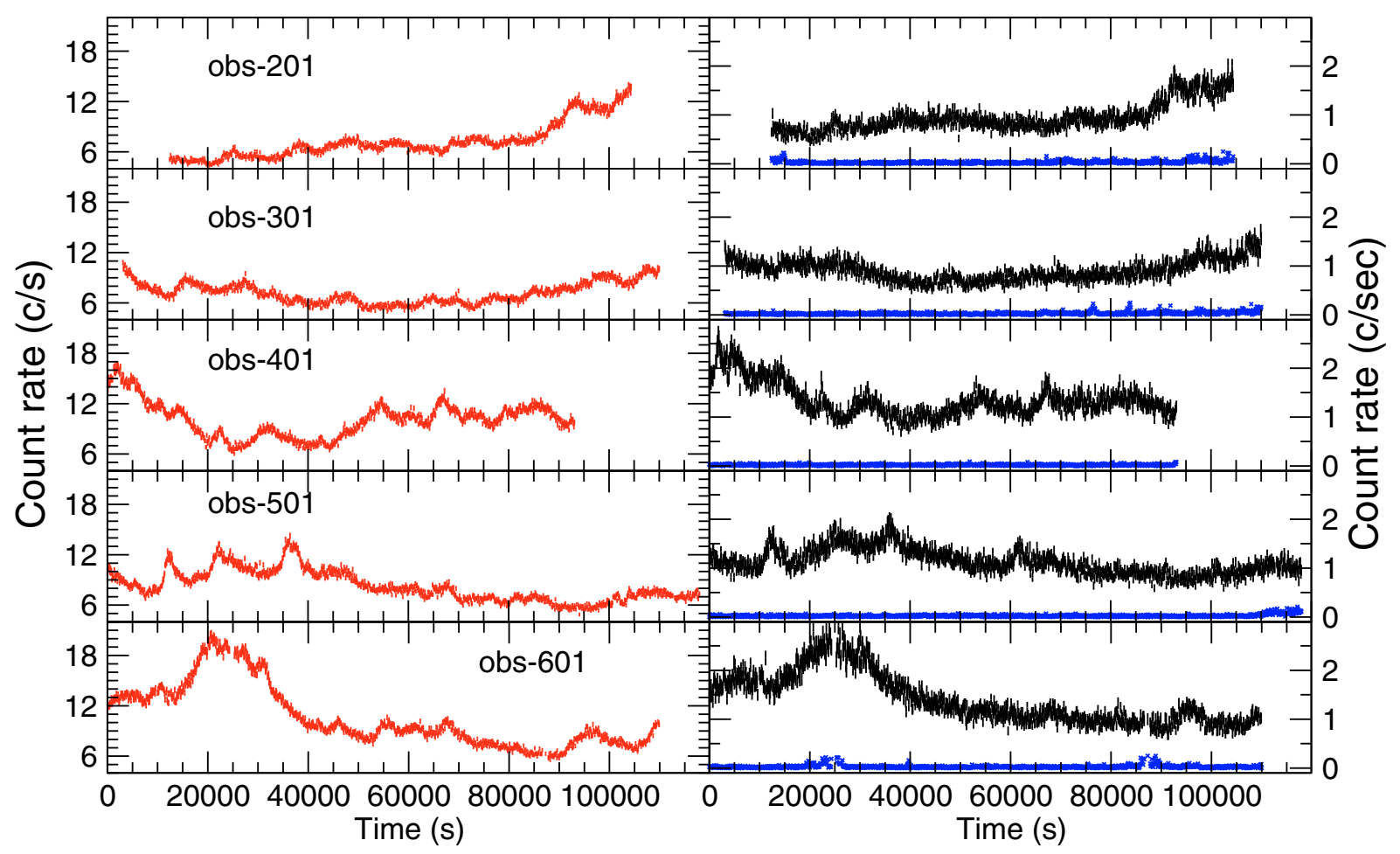

Fig. 1. The PN soft and hard band light curves (left and right panel, respectively) of PKS 0558-504 for all five observations with a binning of $100 \mathrm{~s}$. Time counts from the beginning of each observation. The points plotted with crosses at the bottom of the hard band plots indicate the background count rate in the same energy range.

Array (PCA; Jahoda et al. 1996) and the High-Energy X-Ray Timing Experiment (HEXTE; Rothschild et al. 1998) on RXTE. We used PCA data only, because the signal-to-noise ratio of the HEXTE data is too low for our purposes.

The PCA data were screened according to the following criteria: the satellite had to be out of the South Atlantic Anomaly (SAA) for at least $30 \mathrm{~min}$, the Earth elevation angle was $\geq 10^{\circ}$, the offset from the nominal optical position was $\leq 0.02^{\circ}$, and the parameter ELECTRON-2 was $\leq 0.1$. The last criterion removes data with high particle background rates in the Proportional Counter Units (PCUs). The PCA background light curves were determined using the pca_bkgd_cmfaintl7_eMv20051128.mdl model, which is appropriate for faint sources, and we used the rex script (v.0.3) which runs the FTOOLS program pcabackest. We used PCA, STANDARD-2 mode, 2-10 keV, Layer 1 data, because that is where the PCA is best calibrated and most sensitive. Data were initially extracted with $16 \mathrm{~s}$ time resolution and subsequently re-binned at $5760 \mathrm{~s}$ bins, which is roughly equal to the orbital period of RXTE. PCUs 0 and 2 were turned on throughout the monitoring campaign. However, since the propane layer on PCU0 was damaged in May 2000, causing a systematic increase of the background, we conservatively used only PCU2 for our analysis. All quoted count rates are therefore for one PCU.

Figure 2 shows the long term, background-subtracted 2-10 keV RXTE light curve, binned over one orbit. The data we present in this work span a period of $\sim 4.3$ years. There are variations on all sampled time scales, with a typical amplitude of the order of 1.5-2 over a time scale of 1 week/1 month. The maximum variability amplitude is of the order of $\sim 5$ over just two days. For the PSD analysis we present below, we chose to use those observations which are spaced 2 days apart in the

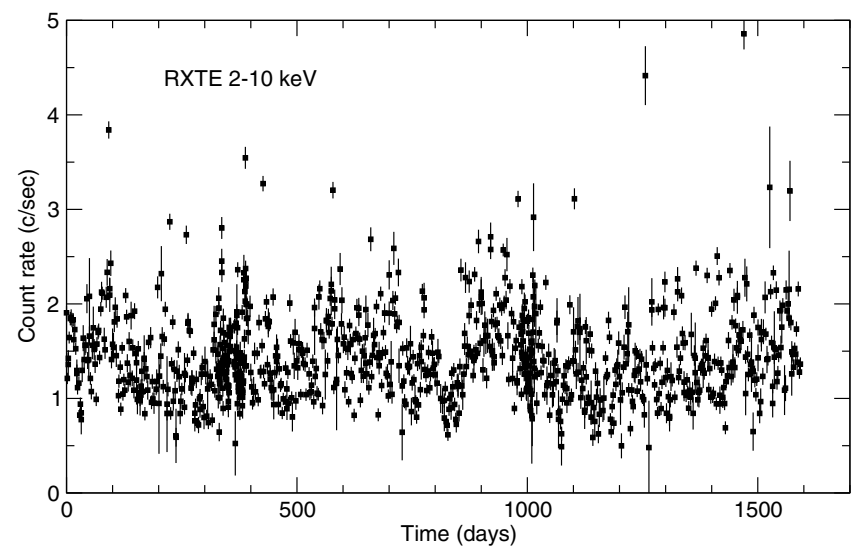

Fig. 2. The long term, 2-10 keV RXTE light curve of PKS 0558-504, with a binning of $5760 \mathrm{~s}$. Time counts in days from the beginning of the first observation.

1-orbit binned light curve. There are 46 points missing, out of 797 points in the resulting light curve. The missing points are spread randomly throughout the total duration of the monitoring campaign. As before with the XMM-Newton light curves, we accounted for them by linear interpolation between the bins adjacent to the gaps, and added the appropriate Poisson noise to the interpolated values.

The duration and sampling interval of the RXTE light curve (in days), its mean count rate and fractional root mean square variability amplitude, are also listed in Table 1 . The error on $F_{\text {var }}$ is $0.3 \%$. The long term variability amplitude of the source is larger than the average amplitude of the XMM-Newton hard band 


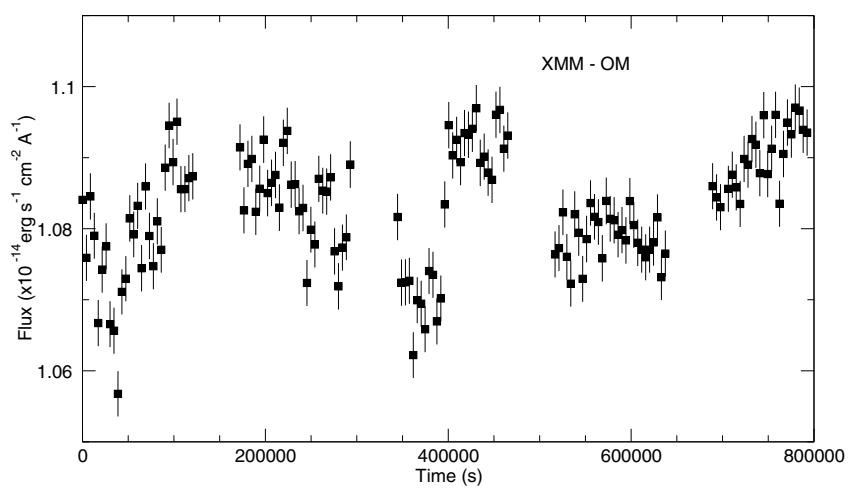

Fig. 3. The $2910 \AA$, OM light curve of PKS 0558-504 with a binning of $4.3 \mathrm{ks}$. The time counts from the beginning of the XMM-Newton observation.

light curves, but this is expected given the much longer duration of the RXTE light curve.

\subsection{The OM observations}

The OM is a $30 \mathrm{~cm}$ optical/UV telescope co-aligned with the $\mathrm{X}$-ray telescopes on board XMM-Newton, which is sensitive in the 1600-6000 $\AA$ wavelength range. For our observations the $\mathrm{OM}$ was operated in imaging mode using the $U V W 1$ filter with an effective wavelength of 291 nanometers. The point spread function in the $U V W 1$ band has a full width at half maximum of $1.7^{\prime \prime}$.

The data consist of a sequence of 140 snapshot exposures, each $4000 \mathrm{~s}$ long, and typically separated by time gaps of $320 \mathrm{~s}$. The source appears point-like on the images. We determined the monochromatic fluxes (erg cm $\mathrm{cm}^{-2} \mathrm{~s}^{-1} \AA^{-1}$ ) for each of the exposures from the pipeline processing results, using the corrected count rates from the "SWSRLI" files according to the recipe given in the corresponding XMM SAS web-site ${ }^{1}$.

The OM light curve is plotted in Fig. 3. Its duration (since the start of the XMM-Newton observations), mean and variability amplitude are listed in Table 1 (the error on $F_{\text {var }}$ is $0.3 \%$ ). The UV source emission is clearly variable. However, the UV variability amplitude is significantly smaller than the variability amplitude of the XMM-Newton single-orbit light curves, despite the fact that the OM light curve plotted in Fig. 3 is $7-8$ times longer than the individual XMM-Newton light curves.

\section{Power spectrum analysis}

\subsection{The high frequency power spectrum}

To estimate the high frequency PSD we used the XMM-Newton soft and hard band light curves of each observation. Following Papadakis \& Lawrence (1993), we calculated the "periodogram" of each light curve (normalized to its mean). Within each energy band, the Poisson noise power level of the light curves is comparable. For this reason, we combined all the soft and all the hard band periodograms in two separate files (one for each band), and sorted them in order of decreasing frequency. We then binned the two "combined periodograms" in log-log space. In order to extend the estimation of the PSD at the lowest possible frequencies, and to increase the frequency "resolution" in the low frequency

\footnotetext{
$1 \mathrm{xmm}$.esac.esa.int/sas/current/watchout/

Evergreen-tips-and-tricks/uvflux.shtml
}

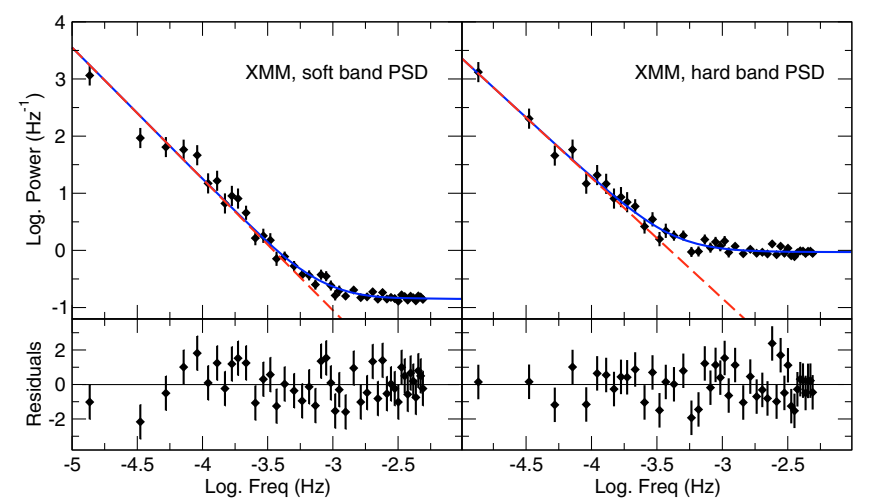

Fig. 4. The soft and hard band power spectra of PKS 0558-504 (left and right panel, respectively). The PSDs have been estimated using the light curves plotted in Fig. 1. The continuous lines indicate the best-fit "PL + Poisson noise" model (dashed lines indicate the PL component of the model). Best-fit residuals are plotted in the bottom panels.

part of the spectrum, we used bins of size 10 for the 10 lowest frequency points, and bins of size $\geq 20$ at higher frequencies.

The soft and hard band PSDs are plotted in Fig. 4 (left and right panel, respectively). They show the familiar "red-noise" shape, similar to what has been observed in other type-1, radioquiet Seyferts. At high frequencies, the PSDs flatten due to the presence of the experimental Poisson noise. The amplitude of this component is larger in the hard band PSD, due to the smaller count rate in this band.

We fitted both PSDs with a power-law model (PL) of the form: $P(f)=A\left(10^{4} f\right)^{-\alpha}+C$, taking into account aliasing effects (as in Papadakis et al. 2003). The constants $A$ and $C$ are the PL normalization (equal to the PSD value at $10^{-4} \mathrm{~Hz}$ ) and the Poisson noise level, respectively. The best fit results are listed in Table 2. The model fits well both spectra. The best-fit, soft-band PSD slope is slighter steeper than the hard-band slope. A similar effect (which is significant is some cases) has been observed in other Seyferts as well.

We followed Papadakis \& Lawrence (1995) to investigate the presence of any significant peaks in the power spectra. We divided the combined, soft and hard band periodograms by the respective best-fit PL models. If a PL model represents well the intrinsic PSD of the source (the "null hypothesis"), the resulting "normalized periodogram estimates", multiplied by 2 , should be distributed as $\chi_{2}^{2}$ variables. Figure 5 shows the soft and hard band "normalized periodogram estimates" plotted for the whole frequency range. The horizontal solid line indicates the expected value of these estimates which, under the null hypothesis, should be equal to 2 . The dashed horizontal line indicates the 95 per cent confidence level (i.e. there is a 5 per cent probability that one of the 2600 normalized periodogram estimates will have a value larger than the value indicated by the dashed lines). No significant peaks are apparent in any part of the spectrum. We therefore conclude that there are no strictly periodic signals in either the soft or hard band XMM-Newton light curves.

\subsection{The case for a QPO in the soft band PSD}

The best-fit soft band PSD residuals in Fig. 4 suggests the presence of a broad excess of power around $\sim 10^{-4} \mathrm{~Hz}$, indicative of the presence of a low-amplitude quasi-periodic oscillation (QPO). No excess of power appears in the hard band PSD at similar frequencies. Gliozzi et al. (2001) and Brinkmann et al. (2004) reported the possible presence of "characteristic" time 
Table 2. PSD model fitting results.

\begin{tabular}{|c|c|c|c|c|c|c|c|c|c|c|}
\hline PSD & Model & $\begin{array}{c}A^{a} \\
\left(\mathrm{~Hz}^{-1}\right)\end{array}$ & $\alpha$ & $\begin{array}{c}C \\
\left(\mathrm{~Hz}^{-1}\right)\end{array}$ & $\begin{array}{c}v_{\mathrm{b}} \\
\left(\times 10^{-6} \mathrm{~Hz}\right)\end{array}$ & $\alpha_{l}$ & $\alpha_{h}$ & $\begin{array}{c}f_{\mathrm{L}} \\
\left(\times 10^{-5} \mathrm{~Hz}\right)\end{array}$ & $\begin{array}{c}R \\
(\%)\end{array}$ & $\chi^{2} /$ d.o.f. \\
\hline$X M M /$ soft & PL & $18_{-3}^{+4}$ & $2.30 \pm 0.14$ & $0.14 \pm 0.01$ & - & - & - & - & - & $43.6 / 42$ \\
\hline$X M M / \mathrm{soft}$ & $\mathrm{PL}+\mathrm{L}$ & $13_{-6}^{+7}$ & $2.24 \pm 0.20$ & $0.14 \pm 0.01$ & - & - & - & $8.2_{-2.1}^{+4.9}$ & $4.9 \pm 2.5$ & $34.9 / 40$ \\
\hline$X M M /$ hard & PL & $19_{-3}^{+4}$ & $2.11 \pm 0.18$ & $0.94 \pm 0.05$ & - & - & - & - & - & $41.2 / 42$ \\
\hline$R X T E+X M M$ & BPL & $64_{-8}^{+24}$ & - & $0.94^{b} / 906^{c}$ & $6.7_{-2.9}^{+4.4}$ & $0.40 \pm 0.17$ & $2.21_{-0.20}^{+0.24}$ & - & - & $51.7 / 53$ \\
\hline
\end{tabular}

Notes. Following Lampton et al. (1976), all errors indicate the 68\% confidence intervals, for 3, 5 and 4 parameters of interest in the case of the PL, BPL and PL+L models, respectively.

(a) PL normalization which is equal to the PSD value at $10^{-4} \mathrm{~Hz}$. In the case of the BPL model, $A$ is defined as in Eq. (2). ${ }^{(b)}$ Poisson noise level for the "high-frequency" part of the PSD. This value is equal to the best-fit value of the PL model fit to the XMM-Newton hard band PSD, and it was kept constant during the model fit. ${ }^{(c)}$ Expected Possion noise level for the low-frequency, RXTE part of the PSD.

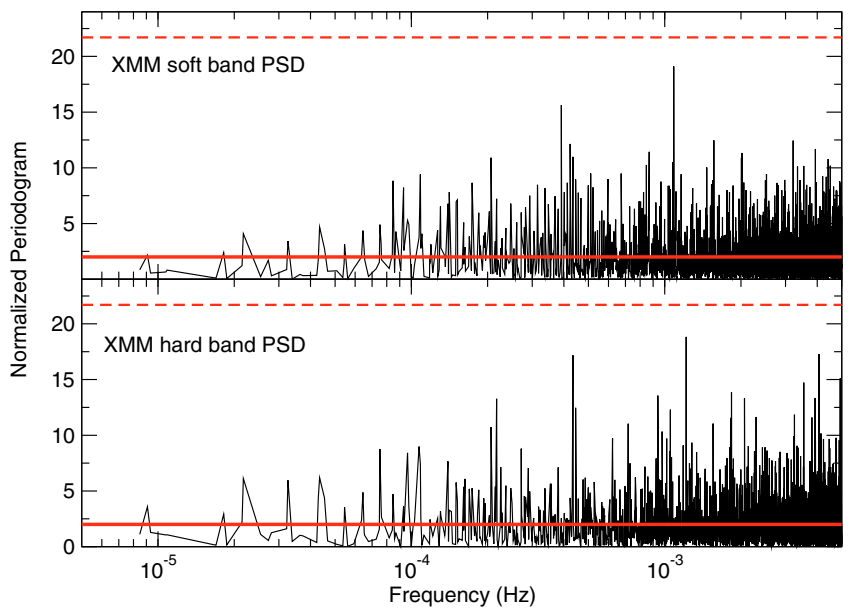

Fig. 5. Plot of the soft and hard band periodograms divided by the respective best-fit PL models (top and bottom panel, respectively). The solid lines indicate the mean value of a $\chi_{2}^{2}$ distributed variable, and the dashed lines the $95 \%$ confidence limits (see text for details).

scales in the source. The first authors relied on the turnover in the structure function to identify a characteristic time scale, but structure functions can show spurious turnovers (due to sampling artifacts for example) even when there are no characteristic time scales in the system (see e.g. Emmanoulopoulos et al. 2010). On the other hand, Brinkmann et al. used several short XMM-Newton observations to identify X-ray flares which seemed to be recurrent with a time scale of $\sim 24 \mathrm{ks}$ but, due to sparse sampling, it was difficult to demonstrate that this signal was indeed significant. Nevertheless, given these reports, and the fact that the search for such time scales was the major aim of the present XMM-Newton observations, we investigate below the possibility that there exists a QPO in the soft band power spectrum.

It is customary to model QPOs in the PSD of Galactic black hole binaries (GBHs) with Lorentzian profiles:

$L(f)=\pi^{-1} \frac{2 R^{2} Q f_{L}}{f_{L}^{2}+4 Q^{2}\left(f-f_{L}\right)^{2}}$,

where $f_{L}$ is the centroid frequency, $Q=f_{L} / \Delta f_{L, F W H M}$ is the quality factor ( $Q \geq 2$ for QPOs), and $R$ is its normalization constant. We therefore re-fitted the soft band PSD with a "PL + a Lorentzian" model (PL+L, hereafter). Initially, the best fit $Q$ value turned out to be $\sim 3$, but with a large uncertainty attached to it. For that reason we repeated the fit with $Q$ kept fixed at 3 .
The model fitted the soft band PSD well. The best-fit PL+L results are listed in Table 2, and the best-fit model is plotted in Fig. 6, together with the residuals plot. The short dashed line indicates the best-fit Lorentzian component.

The $\mathrm{PL}+\mathrm{L}$ model has two more parameters than the PL model, and results in a $\Delta \chi^{2}$ reduction of 8.7. According to the $F$-test, the improvement on the fit when we added the Lorentzian component is significant at $98.8 \%$ confidence. Using a simple likelihood ratio test (e.g. Mushotzky 1982) we reached a similar conclusion: the reduction of the $\chi^{2}$ in the case of the $\mathrm{PL}+\mathrm{L}$ model is significant at $98.2 \%$ confidence. These results imply that the PL $+\mathrm{L}$ model provides a better description of the soft band PSD. However, this does not necessarily "prove" that the peak at $\sim 8 \times 10^{-5} \mathrm{~Hz}$ is significant as well.

One way to investigate this issue is by noticing that, if the QPO is real, Fig. 4 suggests that the excess of power is "spread" over $\sim 30$ periodogram estimates. Although Fig. 5 does not support such a claim, we used the best-fit PL model to normalize the combined soft band periodogram estimates, and we binned neighbouring points in groups of 30, starting from the normalized estimate at the lowest frequency. There are 86 30-binned normalized periodogram estimates, and, due to the additive properties of the $\chi^{2}$ distribution, they should be distributed as $\chi_{60}^{2}$ variables under the null hypothesis. The 95 per cent confidence level for these estimates is 102 , while the value of the second 30-binned estimate (the one that corresponds to the best fit Lorentzian shown in Fig. 6) is 85.1. We therefore conclude that the "QPO" is not statistically significant. Its presence is suggestive, and consistent with the soft band PSD, however it is not required by the data.

\subsection{The broad band power spectrum}

We used the 1-orbit binned RXTE light curve (with the missing points replaced by using linear interpolation, as explained in Sect. 2.3) to estimate the long-term power spectrum of the source. We binned the resulting periodogram using bins of size 10 for the 3 lowest frequency points, and bins of size 40 at higher frequencies. The resulting low-frequency power spectrum is plotted in Fig. 7 (frequencies below $10^{-5} \mathrm{~Hz}$ ). In the same figure we also plot the hard band XMM-Newton PSD (frequencies higher than $10^{-5} \mathrm{~Hz}$ ). The two PSDs combined together can be considered as representative of the $2-10 \mathrm{keV}$ power spectrum of the source over a broad range which covers almost six decades of frequency.

This is correct to the extent that X-ray variability mechanism of the source is stationary (at least over the period of the RXTE 


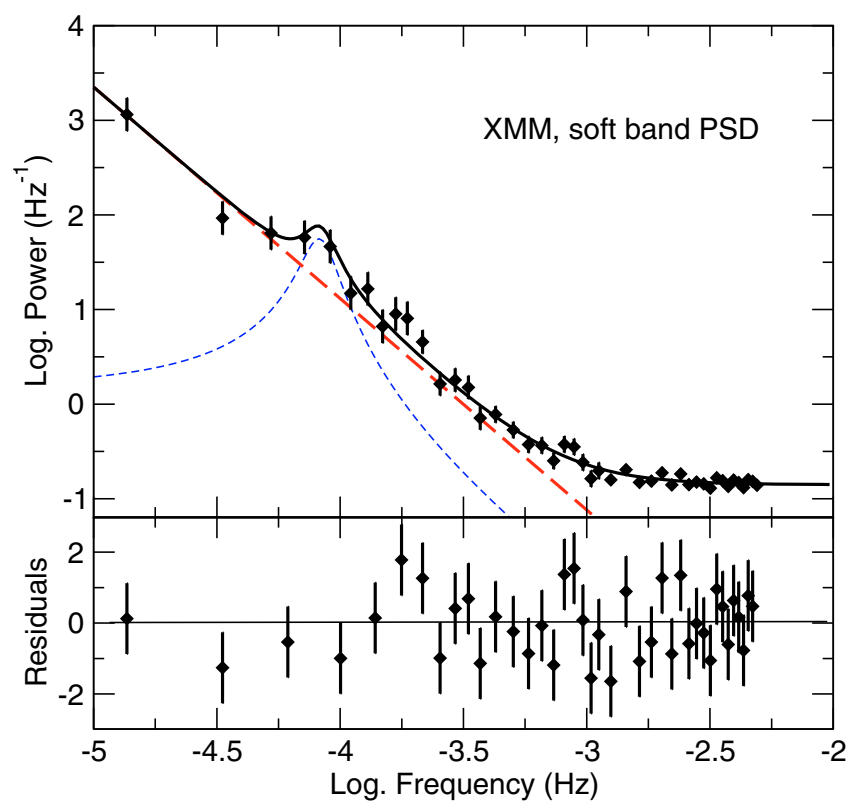

Fig. 6. The soft band power spectrum of PKS 0558-504, plotted together with the best-fit PL+L model (solid line). The long-dashed line indicates the best-fit PL component, and the short-dashed line the best-fit Lorentzian. The best-fit residuals are plotted in the bottom panel.

observations). In addition, the PSD shape may be a function of photon energy in GBHs and AGN. However, significant PSD shape differences are usually detected between the $0.5-2 \mathrm{keV}$ and $2-10 \mathrm{keV}$ energy band, when the difference between the median photon energy in each band is more than 3-4 keV. In our case, the median photon energy of the $2-10 \mathrm{keV} R X T E$ and $X M M$-Newton light curves are $4.9 \mathrm{keV}$ and $3.7 \mathrm{keV}$, respectively. Any PSD shape differences between the two PSDs should therefore be rather small.

Figure 7 clearly shows that the low-frequency power spectrum is significantly flatter than the high-frequency power spectrum. In fact, the combined PSD is reminiscent of the X-ray power spectra of a few type 1 Seyferts which show a " -1 to -2 " frequency "break". The PSD of these sources is well described by a "bending power-law" model (BPL, hereafter), of the form:

$P(v)=A v^{-\alpha_{l}}\left[1+\left(v / v_{b}\right)^{\left(\alpha_{h}-\alpha_{l}\right)}\right]^{-1}$,

where $A$ is the PSD normalization, and $\alpha_{l}, \alpha_{h}$ are the PSD slopes below and above the "break frequency", $v_{b}$ (see e.g. M Mardy et al. 2004). This model fits rather well the power spectrum of the source. Best-fit results are listed in Table 2, while the solid line in Fig. 7 indicates the best-fit model (including aliasing effects and the Poisson noise level for the low and high frequency PSDs). The dashed line in the same figure indicates the best-fit, intrinsic BPL model.

Note that our PSD fitting does not account for "red-noise leakage" that should be present in the estimated power spectra shown in Figs. 4 and 7. However, the amount of red noise leaked power added to the RXTE portion of the combined power spectrum should be small due to the flat spectral slope at low frequencies. As for the high temporal frequency PSDs, the presence of the break just below the minimum sampled temporal frequency means that red noise leaked power should also be small.

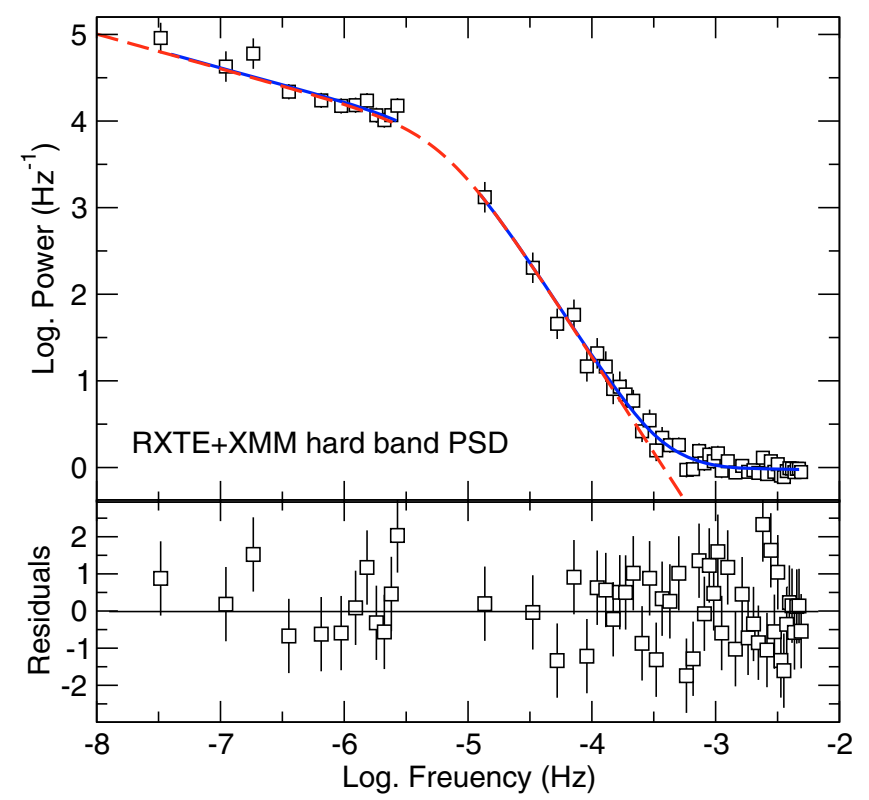

Fig. 7. The combined, 2-10 keV RXTE and XMM-Newton PSDs covering almost 6 decades of frequency. The dashed line indicates the best-fit BPL model to the combined power spectrum. The best-fit residuals are plotted in the bottom panel.

\subsection{The OM power spectrum}

The power spectrum analysis of the OM light curve is not straightforward, due to the small number of OM observations within each individual XMM-Newton observation. For that reason, we considered the OM light curve shown in Fig. 3 as a whole, and we used linear interpolation between the last and the first point of successive observations to fill in the gaps between the five XMM-Newton observations. In this way we created a long, continuous OM light curve from the beginning to the end of the overall XMM-Newton observation. Note that we did not simply add the appropriate Poisson noise in the interpolated values, but the added "noise" was equal to the square root of the total variance of the light curve. The number of missing points in resulting OM light curve is quite large, and corresponds to $24 \%$ of the total number of points.

We binned the OM periodogram using bins of size equal to 13. The OM PSD is plotted Fig. 8 (open diamonds in the lower part of the figure). The OM power spectrum spans a frequency range which is significantly smaller than the frequency range covered by the combined X-ray PSD. Furthermore, given the large number of interpolated data, the OM PSD shape should be somewhat unreliable. For these reasons we did not attempt to fit any models to it. What is important though is that the amplitude of the OM PSD is at least $\sim 1000$ times smaller than the amplitude of the X-ray power spectrum, plotted in Fig. 7.

\section{Summary and discussion}

We present the results from a detailed power spectral analysis of a long XMM-Newton observation (5 orbits in total) of PKS 0558504. We also considered the long RXTE observations of the source ( $\sim 4.3$ years long) to constrain the power spectrum at low frequencies. Our main results can be summarized as follows:

- The high frequency PSDs, in the $0.5-2 \mathrm{keV}$ and $2-10 \mathrm{keV}$ band, have a power-law shape, with a slope of $\sim-2.2$. We did 


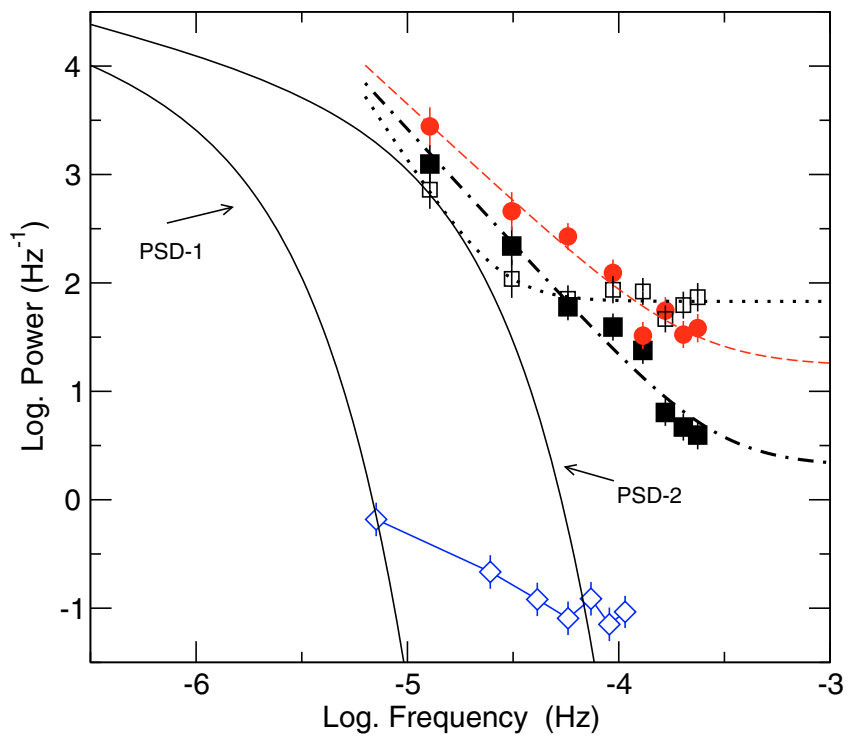

Fig. 8. The OM power spectrum (open diamonds), plotted together with the power spectra of the soft and hard band flux of the power-law component in the source's spectrum (filled circles and filled squares, respectively). Open squares indicate the power spectrum of the "soft-excess" flux light curve (as determined in Paper I). The dashed, dot-dashed and dotted curves indicate the best-fit models to the power spectra (see Sect. 4.3 for details). The solid lines labelled "PSD-1" and "PSD-2" indicate "theoretical" power spectra, as explained in detail in Sect. 4.4.

not find any significant periodic or quasi-periodic signals in the XMM-Newton PSDs.

- The hard band power spectrum has a somewhat flatter slope than the soft band PSD $(2.11 \pm 0.18$ as opposed to $2.30 \pm 0.14)$.

- The broad band, 2-10 keV PSD shows a spectral slope break at a frequency of $\sim 7 \times 10^{-6} \mathrm{~Hz}$, which corresponds to a break time scale, $T_{\mathrm{b}}$, of $\sim 1.7$ days. Best-fit PSD slopes above and below the break are $\sim 2$ and 0.5 , respectively.

- The UV PSD of the source has a significantly smaller amplitude than the X-ray power spectra.

We discuss below in some detail some implications of our results.

\subsection{The "QPO" in the high frequency power spectra}

We found that a Lorentzian-like QPO feature is consistent with the soft band XMM-Newton PSD, but it is not required by the data. The best-fit centroid frequency corresponds to a time scale of $\sim 12000 \mathrm{~s}$, which is half of the "repetition time scale" of flux "peaks" that were detected by Brinkmann et al. (2004) in several XMM-Newton observations of PKS 0558-504. If characteristic frequencies scale in GBHs and AGN with black hole mass, then this QPO, if real, would correspond to a QPO at a frequency of $\sim 800 \mathrm{~Hz}$ for a 10 solar mass $\mathrm{BH}$ (if we assume $M_{\mathrm{BH}} \sim 10^{8} M_{\odot}$ in the case of PKS 0558-504; see below). This would correspond to the so-called "high frequency QPOs" (HFQPOs) which have been detected in the range between 40 and $450 \mathrm{~Hz}$ in a few GBHs. Some of these sources have exhibited single oscillations while others display pairs of HFQPOs with frequencies that scale in a 3:2 ratio (Remillard \& McClintock 2006). Although these oscillations are transient, their centroid frequency does not shift freely in frequency in response to sizeable luminosity changes. Overall, they appear to be stable features that may depend only on the mass and spin of the BH. In fact, their fundamental frequency, $v_{0}$, appears to scale inversely with mass according to the relation $v_{0}(\mathrm{~Hz})=931\left(M_{\mathrm{BH}} / M_{\odot}\right)^{-1}$ (Remillard \& McClintock 2006). This frequency is expected to be $\sim 9 \times 10^{-6} \mathrm{~Hz}$ in the case of a BH with a mass of $\sim 10^{8} M_{\odot}$ (see below). If the QPO-like feature in the soft band PSD corresponds to the stronger QPO (which is usually the one at $2 \times v_{0}$ ) then the frequency of the QPO that should appear in the PSD should be $\sim 2 \times 10^{-5} \mathrm{~Hz}$, which is rather low when compared with the the best-fit centroid frequency listed in Table 2. On the other hand, HFQPOs usually have small amplitudes of $R \sim 1 \%$. This fact could explain the fact why, although a QPO-like feature is suggestive in the soft band PSD, its presence is not statistically significant: a larger number of individual XMM-Newton observations are necessary to detect such a signal with high significance in the PSD of the source.

However, the scaling between the detected HFQPOs in GBHs and the suggestive signal in the soft band PSD of PKS 0558-504 may be coincidental. XMM-Newton light curves allow the PSD estimation for bright AGN in the frequency range between $10^{-5}-10^{-3} \mathrm{~Hz}$. For AGN with $M_{\mathrm{BH}} \sim 10^{6}-10^{8} M_{\odot}$, any non-significant "signal" that will appear by chance in this frequency range, will anyway appear to be related with the HFQPOs in GBHs. We therefore conclude that the evidence for the presence of a intrinsic "QPO-like" feature in the high frequency power spectrum of PKS 0558-504 is not significant.

\subsection{The break frequency in the broad-band $P S D$}

The shape of the broad-band power spectrum of PKS 0558-504 is similar to the power spectral shape of several bright, radioquiet type 1 Seyferts. Their broad-band PSDs have yielded significant evidence for breaks where the PSD slope flattens from $\sim-2$ above the break to $\sim-1$ at frequencies below the break (e.g. $M^{c}$ Hardy et al. 2006). This suggests that the same variability mechanism operates in this source well, and is at odds with the hypothesis of a jet origin for the bulk of the X-ray emission from this object, in agreement with the results of Paper I.

There is no indication of a second PSD break (to a flat slope of $\sim 0$ ) in the broad-band PSD of PKS 0558-504. Ark 564 is the only AGN so far where a second break frequency has been clearly detected in its PSD (Papadakis et al. 2002; $\mathrm{M}^{\mathrm{c}} \mathrm{Hardy}$ et al. 2007). Ark 564 is a high accretion rate source, with a strong "soft-excess" emission in its energy spectrum at energies below $2 \mathrm{keV}$, just like PKS 0558-504. The ratio between the two break frequencies in Ark 564 is $\sim 100$. There is not enough temporal frequency coverage below the break frequency in the PSD of PKS 0558-504 to determine whether there is a second break at frequencies lower than $\sim 10^{-7} \mathrm{~Hz}$ or so. Monitoring covering time scales as long as $\sim 10$ years at least would be needed to determine accurately the low-frequency PSD shape of PKS 0558-504.

$\mathrm{M}^{\mathrm{c}}$ Hardy et al. (2006) have demonstrated that the break time scale $T_{\mathrm{b}}$ in Seyferts scales with $M_{\mathrm{BH}}$, and for a given $\mathrm{BH}$ mass, $T_{\mathrm{b}}$ decreases with increasing bolometric luminosity, $L_{\mathrm{Bol}}: \log \left(T_{\mathrm{b}}\right)$ (days) $=2.1 \log \left(M_{\mathrm{BH}} / 10^{6} M_{\odot}\right)-$ $0.98\left(L_{\mathrm{Bol}} / 10^{44} \mathrm{erg} \mathrm{s}^{-1}\right)-2.32$. The average $2-10 \mathrm{keV}$ luminosity, $L_{2-10 \mathrm{keV}}$, of the source is $\sim 8.4 \times 10^{44} \mathrm{erg} / \mathrm{s}$ (Paper I). Assuming a $\left(L_{\mathrm{Bol}} / L_{2-10 \mathrm{keV})}\right.$ ratio of at least 20 (appropriate for sources with accretion rate larger than 0.1 of the Eddington limit; e.g. Lusso et al. 2010), then $L_{\mathrm{Bol}} \sim 2 \times 10^{46} \mathrm{erg} / \mathrm{s}$. Therefore, the $\mathrm{M}^{\mathrm{c}} \mathrm{Hardy}$ et al. (2006) relationship predicts a BH mass of $\sim 2 \times 10^{8} M_{\odot}$ for PKS 0558-504. This is probably a lower limit on $M_{\mathrm{BH}}$, as 
this source is likely accreting close to the Eddington limit ${ }^{2}$. This estimate is $\sim 3.5$ times larger than the $\mathrm{BH}$ mass estimate we presented in Paper I (based on the virial relationship between $M_{\mathrm{BH}}$, radius and velocity dispersion of the broad line region).

\subsection{The slope of the high-frequency PSD in the soft and hard bands}

Our results indicate that the hard band PSD slope is slightly flatter than the soft band slope. Although the best-fit values are consistent within the $1 \sigma$ uncertainties (hence their difference is not statistically significant). This trend of flatter PSD slopes with increasing energy has been observed in other AGN as well. Papadakis \& Lawrence (1995) were the first to indicate that the soft band $(<2 \mathrm{keV})$ PSD slope was steeper than the hard band ( $>2 \mathrm{keV}$ ) slope in an AGN (namely NGC 4051). This result for NGC 4051 was later confirmed by $\mathrm{M}^{\mathrm{c}} \mathrm{Hardy}$ et al. (2004). A similar trend was also observed in a few more Seyferts (like e.g. Ark 564, Papadakis et al. 2002; MCG -6-30-15, McHardy et al. 2006; Mrk 766, Markowitz et al. 2007).

However, in most cases, a prominent soft-excess emission appears in the energy spectrum of these AGN at energies below $\sim 2 \mathrm{keV}$. This is also the case with PKS 0558-504 (see Paper I). The presence of this component, which could be significantly less variable than the X-ray continuum at energies below $2 \mathrm{keV}$, may dilute the observed variations in the soft band. This effect could explain the steeper power spectrum slope at energies below $2 \mathrm{keV}$. To investigate this issue in more detail, we used the results from the time resolved spectroscopy that we presented in Paper I to study the intrinsic evolution of the PSD slope with energy. The results from this study can be of more general interest as they may apply to Seyferts where the difference between the soft and hard band PSD slopes is significant and which also show a strong, and possibly less variable, soft-excess component at low energies.

To this end, we considered the $0.4-1 \mathrm{keV}$ and $2-10 \mathrm{keV}$ (un-absorbed) flux light curves of the power-law component in the energy spectrum of the source, and the $0.4-1 \mathrm{keV}$ flux of the bremsstrahlung spectral component, which can be considered as as representative of the soft-excess flux in this object (i.e. of the flux of the excess emission on top of the power-law continuum). These light curves have a "bin-size" of $2000 \mathrm{~s}$ (see Paper I, for a detailed description for these light curves). Using the same method that we also used to estimate the soft and hard band XMM-Newton PSDs we estimated the hard and soft band power -law flux PSDs (PSD PL-soft $_{\text {and }}$ PSD $_{\text {PL-hard }}$ ), as well as the soft-excess PSD (PSD $\left.{ }_{\mathrm{SE}}\right)$.

These power spectra are plotted in Fig. 8. The dashed, dotdashed, and dotted lines indicate the best-fit power-law model to $\mathrm{PSD}_{\mathrm{PL}-\text { soft }}, \mathrm{PSD}_{\mathrm{PL}-\text { hard }}$, and $\mathrm{PSD}_{\mathrm{SE}}$ respectively (the constant Poisson noise is also included in the best-fit models plotted in this figure). The best-fit PSD normalization (at $10^{-4} \mathrm{~Hz}$ ) is largest in $\mathrm{PSD}_{\mathrm{PL}-\text { soft }}$ : $70 \pm 35 \mathrm{~Hz}^{-1}$, as compared to $20 \pm 5 \mathrm{~Hz}^{-1}$ and $1.3 \pm 0.5 \mathrm{~Hz}^{-1}$ for the PSD $\mathrm{PL}$-hard and PSD $\mathrm{SE}_{\mathrm{S}}$, respectively. Furthermore, the best-fit PSD $\mathrm{PL}_{\text {-soft }}$, PSD $\mathrm{PL}$-hard, and PSD $_{\mathrm{SE}}$ slopes are $1.8 \pm 0.2,2.1 \pm 0.2$, and $\sim 3$. Although the power spectrum slopes of the power-law continuum in the hard and soft energy bands are consistent within the best-fit uncertainties, the soft band PSD appears now to be flatter than the hard band PSD, and of a higher amplitude. Furthermore, although it is not

\footnotetext{
2 This issue is discussed in detail by Gliozzi et al., submitted to ApJ. The authors used simultaneous XMM-Newton and Swift, as well as radio observations, to get an accurate estimate of its broad-band luminosity.
}

possible to constrain well the slope of the the soft-excess PSD, its amplitude is clearly significantly smaller than the amplitude of both PSD PL-soft $_{\text {and PSD }}$ PL-hard.

This exercise indicates that the apparent power spectral "steepness" of the observed light curve in the soft band is due to the fact that at energies below $2 \mathrm{keV}$, this light curve is the sum of the intrinsic X-ray continuum flux plus the soft-excess flux, which is significantly less variable. As a result, the soft band PSD appears to be steeper (and of lower normalization) when compared with the hard band PSD, while in reality, the PSDs may become steeper, and their normalization may decrease, with increasing energy, in agreement (qualitatively at least) to what is expected if the basic physical mechanism of producing X-rays in PKS 0558-504 involves thermal Comptonisation of soft photons. Although the difference between soft and hard-band power spectrum slopes is not significant in this source, the results we presented above suggest that, even in sources where the PSD slope difference is more pronounced, it may be due to the presence of a less-variable soft-excess component at low energies.

\subsection{The low amplitude OM PSD}

Finally, we comment on the small amplitude of the observed OM variability. As we mentioned above, it is quite likely that PKS 0558-504 operates at a super-Eddington rate. It is also possible that most of the UV emission from AGN which accrete at high rates is dominated by emission from radii between 10-100 Schwarzschild radii $\left(R_{\text {Sch }}\right.$, e.g. Kawaguchi 2003$)$.

It has been suggested in the past (e.g. Lyubarskii 1997) that the emission from a disc region of radius $R$ can follow variations which happen at larger radii, if the time scale of these variations is of the order of, or larger, than $t_{\text {visc }}$ at these radii. For example, if the viscosity fluctuates independently and with the same amplitude, we expect the power spectrum of the disc emission at radius $R$ to have a power-law shape with a slope close to unity over a broad frequency range up to the frequency $\sim 1 / t_{\mathrm{visc}}(R)$. Different power spectrum indices can be obtained if the amplitude of the viscosity variations change with radius.

According to the results from Paper I, the soft-excess emission in PKS 0558-504 below $\sim 2 \mathrm{keV}$ is best explained by a low-temperature Comptonisation component (other alternatives like reflection of the X-ray continuum from an ionized disc are less probable). The Comptonising medium responsible for the soft-excess could be a hot layer on top of the disc, which appears in its innermost region due to the fact that PKS 0558-504 has a large accretion rate which approaches (or even exceeds) the Eddington limit. If both the soft-excess and the OM variations are affected by accretion rate fluctuations which happen at $R>R_{\mathrm{SE}}$ and $R>R_{\mathrm{OM}}$, respectively (where $R_{\mathrm{OM}}$ and $R_{\mathrm{SE}}$ are the radii of the disc regions which contribute most to the softexcess and OM emission), then their power spectra should have a PL shape up to $\sim 1 / t_{\mathrm{visc}}\left(R_{\mathrm{SE}}\right)$ and $\sim 1 / t_{\mathrm{visc}}\left(R_{\mathrm{OM}}\right)$.

The solid curves labelled "PSD-1" and "PSD-2" in Fig. 8 indicate two such PSDs in the case when $R_{\mathrm{OM}}=20 R_{\mathrm{Rsch}}$ and $R_{\mathrm{SE}}=5 R_{\mathrm{Rsch}}$, respectively. At frequencies higher than $1 / t_{\mathrm{visc}}\left(20 R_{\mathrm{Sch}}\right)$ and $1 / t_{\mathrm{visc}}\left(5 R_{\mathrm{Sch}}\right)^{3}$, we have assumed that the PSD decreases exponentially with increasing frequency, like the Cyg X-1 PSD in its high-state (Axelsson et al. 2006), when $\mathrm{X}$-rays are thought to be dominated by emission from the inner

\footnotetext{
3 We have used the Czerny (2006) relationships to estimate the viscous time scales, assuming a viscosity parameter of 0.1 and that the disc thickness over the disc radius ratio is be of the order of unity, as expected in the case of super-Eddington flows.
} 


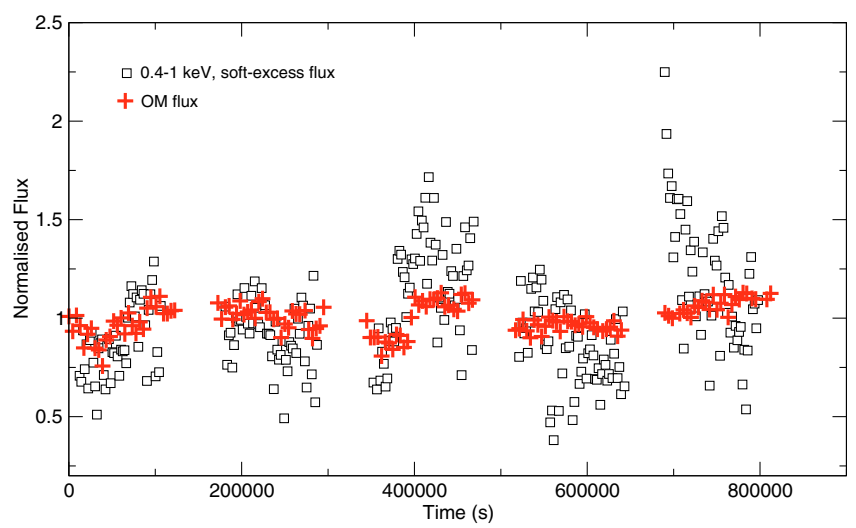

Fig. 9. The OM and soft-excess light curves of the source (crosses and filled squares, respectively). The OM light curve scatter around its mean has been increased by a factor of 10, as explained in Sect. 4.4.

disc region. Both the PSD-1 and PSD-2 curves are consistent with the observed OM and soft-excess power spectra, respectively, although this "agreement" is hard to judge, given the fact that the determination of $\mathrm{PSD}_{\mathrm{OM}}$ and $\mathrm{PSD}_{\mathrm{SE}}$ is poor, even at the lowest observed frequencies.

If indeed the OM and the soft-excess emission originate from the disc at $20 R_{\mathrm{Sch}}$ and $5 R_{\mathrm{Sch}}$, respectively, and their power spectra are like the PSD-1 and PSD-2 curves in Fig. 8, then it is straightforward to show ${ }^{4}$ that the ratio of the excess variance of the soft-excess light curve over the excess variance of the OM light curve should be $\sim 100$. If true, we would expect that $F_{\text {var,SE }} / F_{\text {var,OM }} \sim 10$. The variability amplitude of the softexcess light curve is $\sim 20 \%$ (see Paper I), which implies that the observed ratio of the variability amplitudes $(\sim 20)$, i.e. larger than expected. Nevertheless, a comparison between the soft-excess light curve and the OM light curve, when its amplitude has been multiplied by a factor of 10 , is interesting.

Open squares and crosses in Fig. 9 indicate the soft-excess and OM light curves plotted over the entire period of the $X M M$-Newton observations. We have multiplied the difference of each point in the OM light curve from its mean by 10 , so that the excess variance of the OM light curve increases by a factor of 100. In order to compare the resulting OM light curve with the soft-excess light curve, we divided both of them with their mean.

We note that the soft-excess light curve shows the $0.4-1 \mathrm{keV}$ flux of the bremsstrahlung component that we used in Paper I to parametrise the soft-excess of the source. That is, the points of the soft excess light curve are the result of the time-resolved spectroscopy study we presented in Paper I. The agreement between the observed OM light curve, when scaled by an appropriate factor, and the "calculated" soft-excess light curve is rather good. The soft excess light curve still has a larger variability amplitude, but this is understandable, given the rough estimation of the ratio between the variability amplitude of the two light curves. What is interesting though is that both light curves show the same overall variability patterns during the first 4 XMM-Newton observations.

\footnotetext{
${ }^{4}$ We have assumed that the excess variance of the light curves is approximately equal to the integral of the PSD from the low frequency $1 / T$, where $T \sim 800 \mathrm{ks}$ in this case, i.e. equal to whole duration of the XMM-Newton observations, up to a high frequency $1 /(2 \Delta t)$, where $\Delta t=4.3 \mathrm{ks}$ and $2 \mathrm{ks}$, for the OM and soft-excess light curves, respectively.
}

In fact, this similarity in the variability patterns is somewhat surprising, because the soft excess light curve should be affected by fluctuations in the inner disc, which should not affect the OM light curve. Consequently, we should observe differences not only in the variability amplitudes, but also in the pattern of the observed variations in the two light curves as well. Perhaps a manifestation of this effect is the disagreement of the two light curves during the last XMM-Newton orbit, where the pronounced soft-excess flare at the beginning of the observations is absent in the OM light curve (we note in passing that this flare is also present in the X-ray light curves of the source, and a delay of $\sim 20 \mathrm{ks}$ may exist between the flare in the soft excess and in the soft and hard-band PL flux light curves; see Paper I for details).

Acknowledgements. I.E. and W.P.B. acknowledge support by the EU ToK grant 39965 and FP7-REGPOT 206469. M.G. acknowledges support by the XMM-Newton Guest Investigator Program under NASA grant 201593. This work is based on observations with XMM-Newton, an ESA science mission with instruments and contributions directly funded by ESA Member States and the USA (NASA). This research has made use of the NASA/IPAC Extragalactic Database (NED) which is operated by the Jet Propulsion Laboratory, California Institute of Technology, under contract with the National Aeronautics and Space Administration.

\section{References}

Axelsson, M., Borgonovo, A., \& Larsson, S. 2006, A\&A, 452, 975

Brinkmann, W., Arévalo, P., Gliozzi, M., \& Ferrero, E. 2004, A\&A, 415, 959

Czerny, B. 2006, in AGN Variability from X-Rays to Radio Waves, ed. C. M. Gaskell, I. M. McHardy, B. M. Peterson, \& S. G. Sergeev, ASP Conf. Ser., 360,265

Emmanoulopoulos, D., McHardy, I. M., \& Uttley, P. 2010, MNRAS, 404, 931

Fabian, A. C. 1979, Proc. R. Soc. London, Ser. A, 366, 449

Gliozzi, M., Boller, Th., Brinkmann, W., Brandt, W. A. 2000, A\&A, 356, L17

Gliozzi, M., Brinkmann, W., O’Brien, P. T., et al. 2001, A\&A, 365, L128

Gliozzi, M., Papadakis, I. E., \& Brinkmann, W. 2007, ApJ, 656, 691

Jahoda, K., Swank, J., Giles, A. B., et al. 1996, Proc. SPIE, 2808, 59

Kawaguchi, T. 2003, ApJ, 593, 69

Komossa, S., Voges, W., Xu, D., et al. 2006, AJ, 132, 531

Lampton, M., Margon, B., \& Boyer, S. 1976, ApJ, 208, 177

Lusso, E., Comastri, A., Vignali, C., et al. 2010, A\&A, 512, A34

Lyubarskii, Y. E. 1997, MNRAS, 292, 679

MHardy, I. M., Papadakis, I. E., Uttley, P., Page, M. J., \& Mason, K. O. 2004, MNRAS, 348, 783

McHardy, I. M., Körding, C., Uttley, P., \& Fender, P. P. 2006, Nature, 444, 730

M'Hardy, I. M., Arévalo, P., Uttley, P., et al. 2007, MNRAS, 382, 985

Markowitz, M., Papadakis, I. E., Arévalo, P., et al. 2007, ApJ, 656, 116

Mushotzky, R. 1982, ApJ, 256, 92

Papadakis, I. E., \& Lawrence, A. 1993, MNRAS, 261, 612

Papadakis, I. E., \& Lawrence, A. 1995, MNRAS, 272, 161

Papadakis, I. E., Brinkmann, W., Negoro, H., \& Gliozzi, M. 2002, A\&A, 382, L1

Papadakis, I. E., Reig, P., \& Nandra, K. 2003, MNRAS, 344, 993

Papadakis, I. E., Brinkmann, W., Gliozzi, M., et al. 2010, A\&A, 510, A65

Remillard, R. A., \& McClintock, J. E. 2006, ARAA, 44, 49

Remillard, R. A., Grossan, B., Bradt, H. V., et al. 1991, Nature, 350, 589

Rothschild, R. E., Blanco, P. R., Gruber, D. E., et al. 1998, ApJ, 496, 538

Strüder, L., Briel, U. G., Dennerl, K., et al. 2001, A\&A, 365, L18

Vaughan, S., Fabian, A. C., \& Nandra, K. 2003, MNRAS, 339, 1273

Wang, T., Matsuoka, M., Kubo, H., Mihara, T., \& Negoro, H. 2001, ApJ, 554, 233 